\title{
Generations of monic polynomials such that the coefficients of the polynomials of the next generation coincide with the zeros of the polynomials of the current generation, and new solvable many-body problems
}

\author{
${ }^{*}$ Oksana Bihun ${ }^{1}$ and ${ }^{+}{ }^{\circ}$ Francesco Calogero ${ }^{2}$ \\ *Department of Mathematics, University of Colorado, Colorado Springs, CO, \\ USA \\ +Physics Department, University of Rome "La Sapienza", Italy \\ $\diamond^{\diamond}$ Istituto Nazionale di Fisica Nucleare, Sezione di Roma, Italy \\ 1 obihun@uccs.edu \\ ${ }^{2}$ francesco.calogero@roma1.infn.it, francesco.calogero@uniroma1.it
}

\begin{abstract}
The notion of generations of monic polynomials such that the coefficients of the polynomials of the next generation coincide with the zeros of the polynomials of the current generation is introduced, and its relevance to the identification of endless sequences of new solvable many-body problems "of goldfish type" is demonstrated.
\end{abstract}

Keywords: zeros and coefficients of monic polynomials; generations of monic polynomials; new solvable many-body problems.

Short title: Generations of polynomials and solvable many-body problems

MSC: 70F10, 70K42, 12D99.

\section{Introduction}

Notation 1.1. Unless otherwise indicated, hereafter $N$ is an arbitrary positive integer, $N \geq 2$, indices such as $n, m, \ell, j, \ldots$ run over the integers from 1 to $N$, and superimposed arrows denote $N$-vectors: for instance the vector $\vec{z}$ has the $N$ components $z_{n}$. Exception: hereafter $\vec{\mu}^{(k)}$ is a $k$-vector, with each of its $k$ components $\mu_{1}, \mu_{2}, \ldots, \mu_{k}$ being integers in the range $1 \leq \mu \leq N$ ! (note that this implies that $\vec{\mu}^{(1)} \equiv \mu_{1}$ is a scalar). We use instead a superimposed tilde to denote an unordered set of $N$ numbers: for instance the notation $\tilde{z}$ denotes the unordered set of $N$ numbers $z_{n}$. Upper-case boldface letters denote $N \times N$ matrices: for instance the matrix $\mathbf{M}$ features the $N^{2}$ elements $M_{n m}$. The numbers we use are generally assumed to be complex numbers; except for those restricted to be positive integers (see above), which generally play the role of indices; and "time", see below. The imaginary unit is hereafter denoted as $\mathbf{i}$, implying of course $\mathbf{i}^{2}=-1$. For quantities depending on the real 
independent variable $t$ ("time") superimposed dots indicate differentiation with respect to it: so, for instance, $\dot{z}_{n}(t) \equiv d z_{n}(t) / d t, \ddot{z}_{n} \equiv d^{2} z_{n} / d t^{2}$ (but often the $t$-dependence is not explicitly indicated, whenever this is unlikely to cause any misunderstanding: as, for instance, in the second formula we just wrote). The Kronecker symbol $\delta_{n m}$ has the usual meaning: $\delta_{n m}=1$ if $n=m, \delta_{n m}=0$ if $n \neq m$; and we denote below as $\mathbf{I}$ the unit $N \times N$ matrix the elements of which are $\delta_{n m}$. We adopt throughout the usual convention according to which a void sum vanishes and a void product equals unity: $\sum_{j=J}^{K} f_{j}=0, \prod_{j=J}^{K} f_{j}=1$ if $J>K$. Finally we introduce the following two convenient notations:

$$
\begin{aligned}
& \sigma_{m}(\vec{z})=\sum_{1 \leq s_{1}<s_{2}<\ldots<s_{m} \leq N} z_{s_{1}} z_{s_{2}} \cdots z_{s_{m}} \\
& \sigma_{n, m}(\vec{z})=\delta_{1 m}+\sum_{\substack{1 \leq s_{1}<s_{2}<\ldots<s_{m-1} \leq N \\
s_{j} \neq n, j=1, \ldots, m-1}} z_{s_{1}} z_{s_{2}} \cdots z_{s_{m-1}},
\end{aligned}
$$

where of course the symbol

$$
\sum_{1 \leq s_{1}<s_{2}<\ldots<s_{m} \leq N}
$$

denotes the sum from 1 to $N$ over the $m$ integer indices $s_{1}, s_{2}, \ldots, s_{m}$ with the restriction that $s_{1}<s_{2}<\ldots<s_{m}$, while the symbol

$$
\sum_{\substack{1 \leq s_{1}<s_{2}<\ldots<s_{m-1} \leq N \\ s_{j} \neq n, j=1, \ldots, m-1}}
$$

denotes the sum from 1 to $N$ over the $m-1$ indices $s_{1}, s_{2}, \ldots, s_{m-1}$ with the restriction that $s_{1}<s_{2}<\ldots<s_{m-1}$ and moreover the requirement that all these indices be different from $n$. We note that $\sigma_{n, 1}(\mathbf{z})=1$ according to the convention (see above) that a sum over an empty set of indices equals zero.

Remark 1.1. Note that the notation $\sigma_{m}(\tilde{z})$ (instead of $\sigma_{m}(\vec{z})$ ) is equally meaningful, since this quantity, see (1a), depends only on the symmetric sums of the $N$ components $z_{m}$ of the $N$-vector $\vec{z}$, hence it is independent of the ordering of the $N$ elements $z_{n}$ of the unordered set $\tilde{z}$. The notation $\sigma_{n, m}(\tilde{z})$, instead, is not properly defined and cannot therefore be used; except in the context of expressions which remain valid for any ordering of the $N$ numbers $z_{n}$, i. e., for any assignments of the $N$ different integer labels $n$ (in the range $1 \leq n \leq N$ ) to the $N$ elements of the unordered set $\tilde{z}$; provided of course that the assignment is maintained throughout that expression (in which case the relevant expression amounts in fact to $N$ ! different formulas; assuming, as we generally do, that the $N$ numbers $z_{n}$ are all different among themselves). This remark is of course equally valid for any function $f(\tilde{z})$.

The main protagonists of this paper are the time-dependent monic polynomials of degree $N$ in the variable $z$ :

$$
p_{N}(z ; \vec{y}(t) ; \tilde{x}(t))=z^{N}+\sum_{m=1}^{N}\left[y_{m}(t) z^{N-m}\right]
$$




$$
p_{N}(z ; \vec{y}(t) ; \tilde{x}(t))=\prod_{n=1}^{N}\left[z-x_{n}(t)\right] .
$$

Note that the notation we employ for these polynomials is somewhat redundant, since they are equally well defined by the (time-dependent) $N$-vector $\vec{y}(t)$ the $N$ components of which are the $N$ coefficients $y_{m}(t)$ of the polynomial (see (2a)), as by the (time-dependent) unordered set $\tilde{x}(t)$ the $N$ elements of which are the $N$ zeros $x_{n}(t)$ of the polynomial (see (2b) $)$. Indeed the $N$ coefficients $y_{m}(t)$ can be explicitly expressed in terms of the $N$ zeros $x_{n}(t)$ :

$$
y_{m}=(-1)^{m} \sigma_{m}(\vec{x}) \equiv(-1)^{m} \sigma_{m}(\tilde{x})
$$

(see Notation 1.1 and Remark 1.1). And the $N$ zeros $x_{n}(t)$ are likewise uniquely determined (up to permutations) by the $N$ coefficients $y_{m}(t)$, but of course explicit expressions to this effect are generally available only for $N \leq 4$.

There holds moreover the following identity:

$$
\left(x_{n}\right)^{N}+\sum_{m=1}^{N}\left[y_{m}\left(x_{n}\right)^{N-m}\right]=0
$$

which is an obvious consequence of (2), and via (3) it implies

$$
\left(x_{n}\right)^{N}+\sum_{m=1}^{N}\left[(-1)^{m} \sigma_{m}(\tilde{x})\left(x_{n}\right)^{N-m}\right]=0 .
$$

Note that, while the formula (4a) is an identity valid for the $N$ coefficients $y_{m}$ and the $N$ zeros $x_{n}$ of any polynomial, see (2), the identity (4b) is clearly valid for any arbitrary assignment of the $N$ elements $x_{n}$ of the unordered set $\tilde{x}$.

Likewise, there holds the following formula that is also clearly valid for any assignment of the $N$ elements $x_{n}$ of the unordered set $\tilde{x}$ (see Notation 1.1 and Remark 1.1):

$$
-\left[\prod_{\ell=1, \ell \neq n}^{N}\left(x_{n}-x_{\ell}\right)^{-1}\right] \sum_{j=1}^{N}\left[(-1)^{j}\left(x_{n}\right)^{N-j} \sigma_{m, j}(\tilde{x})\right]=\delta_{n m} ;
$$

note that this formula can also be rewritten in the following $(N \times N)$-matrix version:

$$
\begin{gathered}
{[\mathbf{R}(\tilde{x})]_{n m} \equiv R_{n m}(\tilde{x})=-\left[\prod_{\ell=1, \ell \neq n}^{N}\left(x_{n}-x_{\ell}\right)^{-1}\right]\left(x_{n}\right)^{N-m},} \\
{\left[\mathbf{R}^{-1}(\tilde{x})\right]_{n m} \equiv\left[R^{-1}(\tilde{x})\right]_{n m}=(-1)^{n} \sigma_{n, m}(\tilde{x}),}
\end{gathered}
$$

implying of course (see Notation 1.1 and Remark 1.1)

$$
\mathbf{R}(\tilde{x}) \mathbf{R}^{-1}(\tilde{x})=\mathbf{R}^{-1}(\tilde{x}) \mathbf{R}(\tilde{x})=\mathbf{I} .
$$


Two more identities - the proof of which is elementary, see for instance the Appendix of [7]-relate the time evolution of the $N$ coefficients $y_{m}(t)$ and the $N$ zeros $x_{n}(t)$ of any monic time-dependent polynomial of degree $N$ (see (2), Notation 1.1 and Remark 1.1):

$$
\dot{x}_{n}=-\left[\prod_{\ell=1, \ell \neq n}^{N}\left(x_{n}-x_{\ell}\right)^{-1}\right] \sum_{m=1}^{N}\left(x_{n}\right)^{N-m} \dot{y}_{m},
$$

namely, in vector-matrix form,

$$
\dot{\tilde{x}}=\mathbf{R}(\tilde{x}) \dot{\vec{y}} ;
$$

and

$$
\ddot{x}_{n}=\sum_{\ell=1, \ell \neq n}^{N}\left(\frac{2 \dot{x}_{n} \dot{x}_{\ell}}{x_{n}-x_{\ell}}\right)-\left[\prod_{\ell=1, \ell \neq n}^{N}\left(x_{n}-x_{\ell}\right)^{-1}\right] \sum_{m=1}^{N}\left(x_{n}\right)^{N-m} \ddot{y}_{m} .
$$

And let us report an additional identity which is an obvious consequence of the definitions (3), (1a) and (1b) (see Notation 1.1 and Remark 1.1):

$$
\dot{y}_{m}=(-1)^{m} \quad \dot{\sigma}_{m}(\vec{x}) \equiv(-1)^{m} \sum_{n=1}^{N}\left[\sigma_{n, m}(\tilde{x}) \dot{x}_{n}\right]
$$

or, equivalently (see (5c) and (6b)

$$
\dot{\vec{y}}=\mathbf{R}^{-1}(\tilde{x}) \dot{\tilde{x}} .
$$

In the following Section 2 the notion is introduced of generations of monic polynomials such that the coefficients of the polynomials of the next generation coincide with the zeros of the polynomials of the current generation, while Section 3 indicates how this notion is instrumental in the identification of endless sequences of solvable $N$-body problems. The paper is then concluded by a section entitled "Outlook", where further investigations of the generations of polynomials introduced in Section 2, and of the solvable $N$-body problems identified in Section 3, are outlined.

\section{Generations of monic polynomials}

In this section we introduce the generations of monic polynomials such that the coefficients of the polynomials of the next generation coincide with the zeros of the polynomials of the current generation. This notion does not require that the polynomials under consideration be time-dependent-which will instead be essential for the developments reported in the following section; it instead requires a certain notational generalization in the expression of the polynomials 
via their coefficients and zeros, as shown by the following formulas which generalize expressions (2):

$$
\begin{gathered}
p_{N}^{\left(\vec{\mu}^{(k)}\right)}\left(z ; \vec{y}^{\left(\vec{\mu}^{(k)}\right)} ; \tilde{x}^{\left(\vec{\mu}^{(k)}\right)}\right)=z^{N}+\sum_{m=1}^{N}\left[y_{m}^{\left(\vec{\mu}^{(k)}\right)} z^{N-m}\right], \\
p_{N}^{\left(\vec{\mu}^{(k)}\right)}\left(z ; \vec{y}^{\left(\vec{\mu}^{(k)}\right)} ; \tilde{x}^{\left(\vec{\mu}^{(k)}\right)}\right)=\prod_{n=1}^{N}\left[z-x_{n}^{\left(\vec{\mu}^{(k)}\right)}(t)\right] .
\end{gathered}
$$

Here and below $k$ is a nonnegative integer taking the values $0,1,2, \ldots$, which characterizes the generations of polynomials (as explained below), while (see Notation 1.1) $\vec{\mu}^{(k)}$ is a $k$-vector the $k$ components $\mu_{1}, \mu_{2}, \ldots, \mu_{k}$ of which are integers in the range from 1 to $N$ !, the significance of which is also explained below.

Let us start from the "seed polynomial" characterized by the index $k=0$, for which we use the following notation:

$$
\begin{gathered}
p_{N}^{(0)}\left(z ; \vec{y}^{(0)} ; \tilde{x}^{(0)}\right)=z^{N}+\sum_{m=1}^{N}\left[y_{m}^{(0)} z^{N-m}\right], \\
p_{N}^{(0)}\left(z ; \vec{y}^{(0)} ; \tilde{x}^{(0)}\right)=\prod_{n=1}^{N}\left[z-x_{n}^{(0)}\right] .
\end{gathered}
$$

The polynomials of the first generation $(k=1)$ are then defined as follows (see Notation 1.1):

$$
\begin{gathered}
p_{N}^{\left(\mu_{1}\right)}\left(z ; \vec{y}^{\left(\mu_{1}\right)} ; \tilde{x}^{\left(\mu_{1}\right)}\right)=z^{N}+\sum_{m=1}^{N}\left[y_{m}^{\left(\mu_{1}\right)} z^{N-m}\right], \\
p_{N}^{\left(\mu_{1}\right)}\left(z ; \vec{y}^{\left(\mu_{1}\right)} ; \tilde{x}^{\left(\mu_{1}\right)}\right)=\prod_{n=1}^{N}\left[z-x_{n}^{\left(\mu_{1}\right)}\right],
\end{gathered}
$$

with

$$
\vec{y}^{\left(\mu_{1}\right)}=\vec{x}_{\left[\mu_{1}\right]}^{(0)},
$$

where $\vec{x}_{\left[\mu_{1}\right]}^{(0)}$ is the $N$-vector the $N$ components $x_{\left[\mu_{1}\right], n}^{(0)}$ of which coincide with the $N$ elements of the unordered set $\tilde{x}^{(0)}$ - corresponding to the $N$ zeros of the seed polynomial $p_{N}^{(0)}\left(z ; \vec{y}^{(0)} ; \tilde{x}^{(0)}\right)$, see (10b) - ordered according to their permutation labeled by the specific value of the index $\mu_{1}$ (in the range $1 \leq \mu_{1} \leq N$ !, since there are $N$ ! permutations of the $N$ elements $\left.x_{n}^{(0)}\right)$. Note that this definition (11) of the first-generation (monic) polynomials identifies in fact $N$ ! different polynomials, characterized by the $N$ ! values of the integer index $\mu_{1}$ in the range $1 \leq \mu_{1} \leq N$ !, and that a specific unordered set of zeros $\tilde{x}^{\left(\mu_{1}\right)}$ is associated to each of them, see (11b). 
Remark 2.1. The reader who feels uneasy about the notion that a specific permutation labeled by an index $\mu$ in the range $1 \leq \mu \leq N$ ! identifies a specific order of the $N$ elements $x_{n}$ of an a priori unordered set $\tilde{x}$ may reason as follows. A lexicographic order of the $N$ different complex numbers $x_{n}$ from the unordered set $\left\{x_{1}, \ldots, x_{N}\right\}$ can be used to obtain the first permutation of this unordered set. Recall that the lexicographic rule states that, of two elements with different real parts, the one with algebraically smaller real part comes first, and of two elements with equal real parts, the one with algebraically smaller imaginary part comes first. Once the first permutation $\vec{x}_{[1]}$ of the unordered set $\left\{x_{1}, \ldots, x_{N}\right\}$ is established, one can use the lexicographic order of the permutations of $\vec{x}_{[1]}$ to index, with the index $\mu \in\{1,2, \ldots, N$ ! $\}$, the subsequent $N$ ! permutations of the unordered set $\left\{x_{1}, \ldots, x_{N}\right\}$. Note that for simplicity we always assume to deal with the generic case of (monic) polynomials of degree $N$ featuring $N$ different zeros, and therefore as well $N$ different coefficients because of the way they are defined, as now described, see (11c) and below.

The next, second $(k=2)$ generation of polynomials is then defined as follows (see Notation 1.1):

$$
\begin{gathered}
p_{N}^{\left(\vec{\mu}^{(2)}\right)}\left(z ; \vec{y}^{\left(\vec{\mu}^{(2)}\right)} ; \tilde{x}^{\left(\vec{\mu}^{(2)}\right)}\right)=z^{N}+\sum_{m=1}^{N}\left[y_{m}^{\left(\vec{\mu}^{(2)}\right)} z^{N-m}\right], \\
p_{N}^{\left(\vec{\mu}^{(2)}\right)}\left(z ; \vec{y}^{\left(\vec{\mu}^{(2)}\right)} ; \tilde{x}^{\left(\vec{\mu}^{(2)}\right)}\right)=\prod_{n=1}^{N}\left[z-x_{n}^{\left(\vec{\mu}^{(2)}\right)}\right],
\end{gathered}
$$

with

$$
\vec{y}^{\left(\vec{\mu}^{(2)}\right)}=\vec{x}_{\left[\mu_{2}\right]}^{\left(\mu_{1}\right)}
$$

where $\vec{\mu}^{(2)}=\left(\mu_{1}, \mu_{2}\right)$ and $\vec{x}_{\left[\mu_{2}\right]}^{\left(\mu_{1}\right)}$ is the $N$-vector the $N$ components $x_{\left[\mu_{2}\right], n}^{\left(\mu_{1}\right)}$ of which coincide with the $N$ elements of the unordered set $\tilde{x}^{\left(\mu_{1}\right)}$ - corresponding to the $N$ zeros of the first-generation monic polynomial $p_{N}^{\left(\mu_{1}\right)}\left(z ; \vec{y}^{\left(\mu_{1}\right)} ; \tilde{x}^{\left(\mu_{1}\right)}\right)$, see (11b) - ordered according to their permutation labeled by the specific value of the index $\mu_{2}$ (in the range $1 \leq \mu_{2} \leq N$ !, since there are $N$ ! permutations of the $N$ elements $\left.x_{n}^{\left(\mu_{1}\right)}\right)$. Note that this definition (12) of the second-generation (monic) polynomials identifies in fact $(N !)^{2}$ different polynomials, characterized by the $N$ ! values of the two integer indices $\mu_{1}$ and $\mu_{2}$ (the 2 components of the 2 -vector $\vec{\mu}^{(2)}$ ), each of them in the range from 1 to $N$ !, and that a specific unordered set of zeros $\tilde{x}^{\left(\vec{\mu}^{(2)}\right)}$ is associated to each of these polynomials, see (12b).

It is now clear how all subsequent generations of polynomials are manufactured, all of them being generated by the initial seed polynomial (10). To make the matter completely clear, let us indicate how the $k$-th generation polynomial is defined (see Notation 1.1):

$$
p_{N}^{\left(\vec{\mu}^{(k)}\right)}\left(z ; \vec{y}^{\left(\vec{\mu}^{(k)}\right)} ; \tilde{x}^{\left(\vec{\mu}^{(k)}\right)}\right)=z^{N}+\sum_{m=1}^{N}\left[y_{m}^{\left(\vec{\mu}^{(k)}\right)} z^{N-m}\right]
$$




$$
p_{N}^{\left(\vec{\mu}^{(k)}\right)}\left(z ; \vec{y}^{\left(\vec{\mu}^{(k)}\right)} ; \tilde{x}^{\left(\vec{\mu}^{(k)}\right)}\right)=\prod_{n=1}^{N}\left[z-x_{n}^{\left(\vec{\mu}^{(k)}\right)}\right]
$$

with

$$
\vec{y}^{\left(\vec{\mu}^{(k)}\right)}=\vec{x}_{\left[\mu_{k}\right]}^{\left(\vec{\mu}^{(k-1)}\right)},
$$

where $\vec{\mu}^{(k)}=\left(\vec{\mu}^{(k-1)}, \mu^{k}\right)=\left(\mu_{1}, \ldots, \mu_{k-1}, \mu_{k}\right)$ and $\vec{x}_{\left[\mu_{k}\right]}^{\left(\vec{\mu}^{(k-1)}\right)}$ is the $N$-vector the $N$ components of which coincide with the $N$ elements of the unordered set $\tilde{x}^{\left(\vec{\mu}^{(k-1)}\right)}$ - corresponding to the $N$ zeros of the $(k-1)$-generation monic polynomial $p_{N}^{\left(\vec{\mu}^{(k-1)}\right)}\left(z ; \vec{y}^{\left(\vec{\mu}^{(k-1)}\right)} ; \tilde{x}^{\left(\vec{\mu}^{(k-1)}\right)}\right)$ - ordered according to their permutation labeled by the specific value of the index $\mu_{k}$ (in the range $1 \leq \mu_{k} \leq N$ !, since there are $N$ ! permutations of the $N$ elements $\left.x_{n}^{\left(\vec{\mu}^{(k-1)}\right)}\right)$. Note that this definition (13) of the $k$-generation (monic) polynomials identifies in fact $(N !)^{k}$ different polynomials, characterized by the $N$ ! values of each of the $k$ integer indices $\mu_{1}$, $\mu_{2}, \ldots, \mu_{k}$ (the $k$ components of the $k$-vector $\vec{\mu}^{(k)}$ ), each of them in the range from 1 to $N$ !, and that a specific unordered set of zeros $\tilde{x}^{\left(\vec{\mu}^{(k)}\right)}$ is associated to each of these polynomials, see (13b) (or, equivalently, (9b)).

The first three generations of the monic second-degree polynomials constructed using this procedure, starting from the generic seed polynomial $p_{2}^{(0)}(z)=$ $z^{2}+b z+c$, are provided in Appendix A.

A main motivation for the introduction of these generations of polynomials is because they turn out to be instrumental for the solution of the sequence of new many-body problems defined in the following section. But we submit that - independently from this specific application - this notion deserves further study, given its natural/unnatural character in the context of the theory of polynomials: indeed, a monic polynomial is characterized by its $N$ coefficients as well as by its $N$ zeros, so a sequence of polynomials in which these two sets of numbers exchange sequentially their roles is an intriguing possibility; while interchanging the coefficients and the zeros of polynomials seems unorthodox given the different nature of these two sets of numbers, one ordered and the other one unordered.

\section{Solvable $N$-body problems of goldfish type}

In this section we indicate how to identify endless sequences of solvable $N$-body problems which involve quite naturally the generations of monic polynomials discussed in the preceding section. These $N$-body problems are characterized by equations of motion of Newtonian type ("acceleration equals force"), describing the motion in the complex $z$-plane of $N$ unit-mass point-particles interacting among themselves with prescribed forces depending on their positions and their velocities. The prototypical example is the so-called "goldfish" $N$-body model 
(for the name see [4]), characterized by the equations of motion

$$
\ddot{x}_{n}=\sum_{\ell=1, \ell \neq n}^{N}\left(\frac{2 \dot{x}_{n} \dot{x}_{\ell}}{x_{n}-x_{\ell}}\right) \text {. }
$$

A simple generalization of these equations of motion (14), featuring the arbitrary parameter $\omega$ (and reducing to (14) for $\omega=0$ ), reads as follows:

$$
\ddot{x}_{n}=\mathbf{i} \omega \dot{x}_{n}+\sum_{\ell=1, \ell \neq n}^{N}\left(\frac{2 \dot{x}_{n} \dot{x}_{\ell}}{x_{n}-x_{\ell}}\right) \text {. }
$$

The solution of the corresponding initial-values problem is provided by the $N$ roots $x_{n} \equiv x_{n}(t)$ of the following, rather neat, single algebraic equation in the variable $z$,

$$
\sum_{\ell=1, \ell \neq n}^{N}\left[\frac{\dot{x}_{\ell}(0)+\mathbf{i} \omega x_{\ell}(0)}{z-x_{\ell}(0)}\right]=\frac{\mathbf{i} \omega}{\exp (\mathbf{i} \omega t)-1},
$$

which is actually a polynomial equation of degree $N$ in $z$, as seen after multiplication by the product $\prod_{n=1}^{N}\left[z-x_{n}(0)\right]$. Hence- whenever the parameter $\omega$ is real and nonvanishing, as hereafter assumed - this model is isochronous: all its solutions are completely periodic, with the period $T=2 \pi /|\omega|$ (see the function in the right-hand side of (15b) ); or possibly, due to an exchange of the particle positions after the time $T$, with a period which is a (generally small: see [1]) integer multiple of $T$.

Several solvable generalizations of the goldfish model, characterized by Newtonian equations of motion featuring additional forces besides those appearing in the right-hand side of (14), are known: see for instance [3], the two books [5] 6] and references therein, the quite recent papers [7] [2] [8], and the entry "goldfish many-body problem" in Google or Google Scholar.

Above and hereafter an $N$-body model is considered solvable if the configuration of the system at any arbitrary time $t$ can be obtained-for given initial data: the initial positions and velocities of the $N$ particles in the complex $z$ plane-by algebraic operations, such as finding the $N$ zeros $x_{n}(t)$ of an explicitly known time-dependent polynomial of degree $N$ in $z$ (of course such an algebraic equation can be explicitly solved only for $N \leq 4$ ).

Remark 3.1. Note however that knowledge of the configuration of the $N$-body system at time $t$-i. e., of the (generally complex) values of the $N$ coordinates $x_{n}(t)$ given as the unordered set of the $N$ zeros of a known polynomial of degree $N$ in $z$-does not allow to identify the specific coordinate, say, $x_{1}(t)$ that has evolved over time from the specific initial data $x_{1}(0), \dot{x}_{1}(0)$; this additional information can only be gained by following over time the evolution of the system, either by integrating numerically the equations of motion, or by identifying the configurations of the system (as given by the $N$ zeros of a polynomial) at a sequence of time intervals sufficiently close to each other so as to guarantee the identification by continuity of the trajectory of each particle (or 
at least of the specific particle under consideration). Note however that these additional operations need not be performed with great accuracy, even when one wishes the final configuration - including the identity of each particle - to be known with much greater accuracy.

Likewise - in the case of systems which have been identified as isochronous because their solution is provided by the $N$ zeros $x_{n}(t)$ of a time-dependent polynomial of degree $N$ in $z$ which is itself periodic in time with period, say, $T$ (as in the above example, see (15)) - an analogous procedure must be followed to ascertain whether the period of the time evolution of a specific particle is $T$, or $p T$ (with $p$ a positive integer certainly not larger than $N$ !; indeed, generally much smaller, see [11]), due to a periodic exchange of the correspondence between the zeros of the polynomial and the particle identities.

The key formulas for the following developments are the identities (7) and (8), relating the time evolution of the coefficients $x_{n}(t)$ of the zeros of a timedependent (monic) polynomial to that of the coefficients $y_{m}(t)$ of the same polynomial, as well as the notion of generations of polynomials discussed in the previous Section 2.

The starting point of our treatment is any one of the many known solvable $N$-body models - see for instance [5] [6] and the literature therein, and below for an example - characterized by (Newtonian) equations of motion which we write as follows (see Remark 1.1):

$$
\ddot{x}_{n}=f_{n}(\tilde{x}, \dot{\tilde{x}})=f_{n}(\vec{x}, \dot{\vec{x}}) .
$$

We then consider the generations of (time-dependent) polynomials of degree $N$ in $z$ originating from the (time-dependent) seed polynomial

$$
\begin{gathered}
p_{N}^{(0)}\left(z ; \vec{y}^{(0)}(t) ; \tilde{x}^{(0)}(t)\right)=z^{N}+\sum_{m=1}^{N}\left[y_{m}^{(0)}(t) z^{N-m}\right], \\
p_{N}^{(0)}\left(z ; \vec{y}^{(0)}(t) ; \tilde{x}^{(0)}(t)\right)=\prod_{n=1}^{N}\left[z-x_{n}^{(0)}(t)\right],
\end{gathered}
$$

the (time-dependent) zeros $x_{n}^{(0)}(t)$ of which are the solution of the solvable model (16):

$$
x_{n}^{(0)}(t)=x_{n}(t) .
$$

It is then plain via (7) that the zeros $x_{n}^{\left(\mu_{1}\right)}(t)$ of the first generation polynomials (see (11c) and (17c))

$$
\begin{gathered}
p_{N}^{\left(\mu_{1}\right)}\left(z ; \vec{x}_{\left[\mu_{1}\right]}(t) ; \tilde{x}^{\left(\mu_{1}\right)}(t)\right)=z^{N}+\sum_{m=1}^{N}\left[x_{\left[\mu_{1}\right], m}(t) z^{N-m}\right], \\
p_{N}^{\left(\mu_{1}\right)}\left(z ; \vec{x}_{\left[\mu_{1}\right]}(t) ; \tilde{x}^{\left(\mu_{1}\right)}(t)\right)=\prod_{n=1}^{N}\left[z-x_{n}^{\left(\mu_{1}\right)}(t)\right],
\end{gathered}
$$


provide the solutions of the $N$-body problems

$$
\begin{aligned}
& \ddot{x}_{n}^{\left(\mu_{1}\right)}=\sum_{\ell=1, \ell \neq n}^{N}\left(\frac{2 \dot{x}_{n}^{\left(\mu_{1}\right)} \dot{x}_{\ell}^{\left(\mu_{1}\right)}}{x_{n}^{\left(\mu_{1}\right)}-x_{\ell}^{\left(\mu_{1}\right)}}\right) \\
& -\left[\prod_{\ell=1, \ell \neq n}^{N}\left(x_{n}^{\left(\mu_{1}\right)}-x_{\ell}^{\left(\mu_{1}\right)}\right)^{-1}\right] \sum_{m=1}^{N}\left(x_{n}^{\left(\mu_{1}\right)}\right)^{N-m} f_{m}\left(\vec{y}^{\left(\mu_{1}\right)}, \dot{\vec{y}}^{\left(\mu_{1}\right)}\right)
\end{aligned}
$$

with, in the right hand side, the components of the $N$-vectors $\vec{y}^{\left(\mu_{1}\right)} \equiv \vec{y}^{\left(\mu_{1}\right)}(t)$ and $\dot{\vec{y}}^{\left(\mu_{1}\right)} \equiv d \vec{y}^{\left(\mu_{1}\right)}(t) / d t$ replaced of course by their expressions in terms of the components $x_{\left[\mu_{1}\right], n}(t)$ respectively $\dot{x}_{\left[\mu_{1}\right], n}(t)$ of the $N$-vectors $\vec{x}_{\left[\mu_{1}\right]}(t)$ respectively $d \vec{x}_{\left[\mu_{1}\right]}(t) / d t$ as follows:

$$
\begin{gathered}
y_{m}^{\left(\mu_{1}\right)}=(-1)^{m} \sigma_{m}\left(\vec{x}_{\left[\mu_{1}\right]}\right) \\
\dot{y}_{m}^{\left(\mu_{1}\right)}=(-1)^{m} \sum_{n=1}^{N}\left[\sigma_{n, m}\left(\vec{x}_{\left[\mu_{1}\right]}\right) \dot{x}_{n}^{\left(\mu_{1}\right)}\right]
\end{gathered}
$$

(see (3) and (8)). Note that in this manner we have identified $N$ ! new $N$-body problems, labeled by the index $\mu_{1}$ taking integer values in the range from 1 to $N$ ! (the significance of which is explained in Section 2), and characterized by the Newtonian equations of motion of goldfish type (19). These new $N$-body problems are of course solvable, since their solutions are provided by the zeros of the polynomials (18) which are known because their coefficients are (a given permutation, characterized by the index $\mu_{1}$, of) the solutions of the problem (16), which is assumed to begin with to be itself solvable.

And it is now plain how this technique can be iterated over and over again in order to identify new solvable $N$-body problems. Let us just exhibit-relying on the notation of Section 2 - the $(N !)^{2}$ solvable $N$-body problems yielded by the first iteration of this procedure. The corresponding Newtonian equations of motion of goldfish type read as follows:

$$
\begin{aligned}
& \ddot{x}_{n}^{\left(\vec{\mu}^{(2)}\right)}=\sum_{\ell=1, \ell \neq n}^{N}\left(\frac{2 \dot{x}_{n}^{\left(\vec{\mu}^{(2)}\right)} \dot{x}_{\ell}^{\left(\vec{\mu}^{(2)}\right)}}{x_{n}^{\left(\vec{\mu}^{(2)}\right)}-x_{\ell}^{\left(\vec{\mu}^{(2)}\right)}}\right) \\
& -\left[\prod_{\ell=1, \ell \neq n}^{N}\left(x_{n}^{\left(\vec{\mu}^{(2)}\right)}-x_{\ell}^{\left(\vec{\mu}^{(2)}\right)}\right)^{-1}\right] \sum_{m=1}^{N}\left(x_{n}^{\left(\vec{\mu}^{(2)}\right)}\right)^{N-m} \ddot{y}_{m}^{\left(\vec{\mu}^{(2)}\right)},
\end{aligned}
$$

where $\vec{\mu}^{(2)}$ is the 2 -vector with two components $\mu_{1}$ and $\mu_{2}$ (each of them taking integer values from 1 to $N$ !). For each of the $(N !)^{2}$ assignment of this 2 -vector $\vec{\mu}^{(2)}$ (the significance of which has been explained in Section 2), this set of $N$ Newtonian equations of motion of goldfish type determine the time-evolution of 
the coordinates $x_{n}^{\left(\vec{\mu}^{(2)}\right)} \equiv x_{n}^{\left(\vec{\mu}^{(2)}\right)}(t)$, with the quantity $\ddot{y}_{m}^{\left(\vec{\mu}^{(2)}\right)}$ appearing in the right-hand side of (20a) being replaced as follows:

$$
\begin{aligned}
& \ddot{y}_{m}^{\left(\vec{\mu}^{(2)}\right)}=\sum_{\ell=1, \ell \neq n}^{N}\left(\frac{2 \dot{y}_{m}^{\left(\vec{\mu}^{(2)}\right)} \dot{y}_{\ell}^{\left(\vec{\mu}^{(2)}\right)}}{y_{m}^{\left(\vec{\mu}^{(2)}\right)}-y_{\ell}^{\left(\vec{\mu}^{(2)}\right)}}\right) \\
& -\left[\prod_{\ell=1, \ell \neq m}^{N}\left(y_{m}^{\left(\vec{\mu}^{(2)}\right)}-y_{\ell}^{\left(\vec{\mu}^{(2)}\right)}\right)^{-1}\right] \sum_{n=1}^{N}\left(y_{m}^{\left(\vec{\mu}^{(2)}\right)}\right)^{N-n} f_{n}\left(\vec{y}^{\left(\vec{\mu}^{(2)}\right)}, \dot{\vec{y}}^{\left(\vec{\mu}^{(2)}\right)}\right),
\end{aligned}
$$

where moreover, in the right-hand side of this expression, the quantities $y_{m}^{\left(\vec{\mu}^{(2)}\right)} \equiv$ $y_{m}^{\left(\vec{\mu}^{(2)}\right)}(t)$ and $\dot{y}_{m}^{\left(\vec{\mu}^{(2)}\right)}=\dot{y}_{m}^{\left(\vec{\mu}^{(2)}\right)}(t)$ should be replaced by their expressions in terms of the coordinates $x_{n}^{\left(\vec{\mu}^{(2)}\right)} \equiv x_{n}^{\left(\vec{\mu}^{(2)}\right)}(t)$ and their time-derivatives $\dot{x}_{n}^{\left(\vec{\mu}^{(2)}\right)} \equiv$ $\dot{x}_{n}^{\left(\vec{\mu}^{(2)}\right)}(t)$, as follows (see again (3) and (마) ):

$$
\begin{gathered}
y_{m}^{\left(\vec{\mu}^{(2)}\right)}=(-1)^{m} \sigma_{m}\left(\vec{x}^{\left(\vec{\mu}^{(2)}\right)}\right), \\
\dot{y}_{m}^{\left(\vec{\mu}^{(2)}\right)}=(-1)^{m} \sum_{n=1}^{N}\left[\sigma_{n, m}\left(\vec{x}^{\left(\vec{\mu}^{(2)}\right)}\right) \dot{x}_{n}^{\left(\vec{\mu}^{(2)}\right)}\right] .
\end{gathered}
$$

As a particularly simple example, let us display the solvable $N$-body problems written above, corresponding to the assignment (with $a$ an arbitrary constant)

$$
f_{n}(\vec{x}, \dot{\vec{x}})=(\mathbf{i}-a) \dot{x}_{n}-\mathbf{i} a x_{n},
$$

implying (see (16))

$$
x_{n}(t)=\frac{\left[a x_{n}(0)+\dot{x}_{n}(0)\right] \exp (\mathbf{i} t)+\left[\mathbf{i} x_{n}(0)-\dot{x}_{n}(0)\right] \exp (-a t)}{\mathbf{i}+a} .
$$

Then the first generation of solvable $N$-body problems is characterized by the following Newtonian equations of motion of goldfish type (see (19))

$$
\begin{aligned}
& \ddot{x}_{n}^{\left(\mu_{1}\right)}=\sum_{\ell=1, \ell \neq n}^{N}\left(\frac{2 \dot{x}_{n}^{\left(\mu_{1}\right)} \dot{x}_{\ell}^{\left(\mu_{1}\right)}}{x_{n}^{\left(\mu_{1}\right)}-x_{\ell}^{\left(\mu_{1}\right)}}\right) \\
& -\left[\prod_{\ell=1, \ell \neq n}^{N}\left(x_{n}^{\left(\mu_{1}\right)}-x_{\ell}^{\left(\mu_{1}\right)}\right)^{-1}\right] \sum_{m=1}^{N}\left(x_{n}^{\left(\mu_{1}\right)}\right)^{N-m}\left[(\mathbf{i}-a) \dot{y}_{m}^{\left(\mu_{1}\right)}-\mathbf{i} a y_{m}^{\left(\mu_{1}\right)}\right]
\end{aligned}
$$


which, via (6a) and (4a), may in this case be simplified to read

$$
\begin{aligned}
& \ddot{x}_{n}^{\left(\mu_{1}\right)}=\sum_{\ell=1, \ell \neq n}^{N}\left(\frac{2 \dot{x}_{n}^{\left(\mu_{1}\right)} \dot{x}_{\ell}^{\left(\mu_{1}\right)}}{x_{n}^{\left(\mu_{1}\right)}-x_{\ell}^{\left(\mu_{1}\right)}}\right)+(\mathbf{i}-a) \dot{x}_{n}^{\left(\mu_{1}\right)} \\
& -\mathbf{i} a\left[\prod_{\ell=1, \ell \neq n}^{N}\left(x_{n}^{\left(\mu_{1}\right)}-x_{\ell}^{\left(\mu_{1}\right)}\right)^{-1}\right]\left(x_{n}^{\left(\mu_{1}\right)}\right)^{N} .
\end{aligned}
$$

While the equations of motion of the Newtonian equations of motion of goldfish type characterizing the second generation of solvable $N$-body problems read (see (201)

$$
\begin{aligned}
& \ddot{x}_{n}^{\left(\vec{\mu}^{(2)}\right)}=\sum_{\ell=1, \ell \neq n}^{N}\left(\frac{2 \dot{x}_{n}^{\left(\vec{\mu}^{(2)}\right)} \dot{x}_{\ell}^{\left(\vec{\mu}^{(2)}\right)}}{x_{n}^{\left(\vec{\mu}^{(2)}\right)}-x_{\ell}^{\left(\vec{\mu}^{(2)}\right)}}\right) \\
& -\left[\prod_{\ell=1, \ell \neq n}^{N}\left(x_{n}^{\left(\vec{\mu}^{(2)}\right)}-x_{\ell}^{\left(\vec{\mu}^{(2)}\right)}\right)^{-1}\right] \sum_{m=1}^{N}\left(x_{n}^{\left(\vec{\mu}^{(2)}\right)}\right)^{N-m} \ddot{y}_{m}^{\left(\vec{\mu}^{(2)}\right)},
\end{aligned}
$$

with the quantity $\ddot{y}_{m}^{\left(\vec{\mu}^{(2)}\right)}$ appearing in the right-hand side of (23a) being replaced as follows:

$$
\begin{aligned}
& \ddot{y}_{m}^{\left(\vec{\mu}^{(2)}\right)}=\sum_{\ell=1, \ell \neq n}^{N}\left(\frac{2 \dot{y}_{m}^{\left(\vec{\mu}^{(2)}\right)} \dot{y}_{\ell}^{\left(\vec{\mu}^{(2)}\right)}}{y_{m}^{\left(\vec{\mu}^{(2)}\right)}-y_{\ell}^{\left(\vec{\mu}^{(2)}\right)}}\right)+(\mathbf{i}-a) \dot{y}_{m}^{\left(\vec{\mu}^{(2)}\right)} \\
& -\mathbf{i} a\left[\prod_{\ell=1, \ell \neq m}^{N}\left(y_{m}^{\left(\vec{\mu}^{(2)}\right)}-y_{\ell}^{\left(\vec{\mu}^{(2)}\right)}\right)^{-1}\right]\left(y_{m}^{\left(\vec{\mu}^{(2)}\right)}\right)^{N},
\end{aligned}
$$

of course with the additional replacements (20c) and (20d).

Remark 3.2. Clearly the original $N$-body system of this example, see (16) with (21), has the property to be isochronous (with period $2 \pi$ ) if $a=0$, and to be asymptotically isochronous if the parameter $a$ is an arbitrary positive number (for the notion of asymptotic isochrony see [9] and Chapter 6 of [6]). And it is plain that these features are then preserved by all the solvable $N$-body problems generated from this original model (of which the first two instances are exhibited above); and also that this property - the inheritance of isochrony or asymptotic isochrony, as the case may be, by all $N$-body problems generated by an original model possessing these features - is a general characteristic of the class of solvable systems generated by an iteration of the procedure based on the repeated use of the identity (7).

Several new solvable $N$-body problems generated by this technique-but only restricted to the first application of the identity (7), without any further 
iteration - have been already investigated: see [7] [2] [8]. In the present paper we limit our presentation to the general introduction of this technique - which has clearly the potential to yield endless sequences of solvable systems - and to the single example of its application including just one iteration, as described above. Specific discussion of other such models - as well as more complete analyses of the behavior of the solutions of the new models discussed in the recent papers we just quoted - might be undertaken by ourselves or others to the extent that these findings evoke sufficient interest.

Let us end this section with the following

Remark 3.3. It is clear from our treatment that the endless sequences of solvable $N$-body problems of goldfish type associated via the technique described above to any solvable seed problem are in fact yielded by an appropriate sequence of changes of dependent variables. It might therefore be concluded that all these models are, as it were, trivially equivalent to the original seed model. But such an opinion would clash with the fact that most-perhaps all-the solvable $N$-body models which have been identified and investigated worldwide in the last few decades - their discovery and analysis constituting a substantial development of mathematics and mathematical physics over the last few decades - are as well reducible to altogether trivial time evolutions by appropriate changes of dependent variables. The rub is the identification-and the investigation - of the appropriate changes of dependent variables; namely, in the present context, further study of the notion of generations of polynomials as described in Section 2.

\section{Outlook}

The findings reported in this paper suggest further developments, which ourselves or others will hopefully pursue and report in future publications.

Investigation of the properties of the zeros of the polynomials belonging to subsequent generations as defined in Section 2 - and of the associated Riemann surfaces - is a topic that naturally belongs to algebraic geometry, and which does not seem to have been investigated so far (to the best of our knowledge), while, in our opinion, it deserves further study; as well as other properties of these polynomials, for instance when they originate from "named" polynomial seeds.

We originally believed that Diophantine properties of the zeros of the polynomials belonging to the generations of polynomials yielded, say, by one of the classical polynomials playing the role of seed, could be obtained by an appropriate investigation of appropriate dynamical systems - of the type discussed in Section 3- in the immediate vicinity of their equilibria; indeed this is one of the techniques - see for instance [1] or [5] [6] and references therein - that allows to show, for instance, that the $(N \times N)$-matrix $\mathbf{M}(\tilde{x})$ defined componentwise as follows in terms of the zeros $x_{n}$ of the Hermite polynomial $H_{N}(z)$ (see for 
instance [10])

$$
\begin{gathered}
M_{n m}(\tilde{x})=-\left(x_{n}-x_{m}\right)^{-2}, \quad n \neq m, \\
M_{n n}(\tilde{x})=\sum_{\ell=1, \ell \neq n}^{N}\left(x_{n}-x_{\ell}\right)^{-2}=-\sum_{\ell=1, \ell \neq n}^{N} M_{n \ell}(\tilde{x}),
\end{gathered}
$$

features the $N$ eigenvalues $0,1, \ldots, N-1$. (Note that these formulas define in fact $N$ ! different matrices due to the unordered character of the set $\tilde{x}$, but these matrices are related to each other by a permutation of their $N$ lines and a corresponding permutation of their $N$ columns, so that the fact that they all feature the same spectrum is automatically guaranteed). And indeed we were able to identify in this manner $(N \times N)$-matrices $\mathbf{M}^{\left(\mu_{1}\right)}\left(\tilde{x}, \tilde{x}^{\left(\mu_{1}\right)}\right)$ which feature the same eigenvalues $0,1, \ldots, N-1$, being defined in terms of the $N$ zeros $x_{n}$ of the Hermite polynomial $H_{N}(z)$ and of the $N$ zeros $x_{n}^{\left(\mu_{1}\right)}$ of each of the first generation of polynomials yielded, via the technique of Section 2, by the Hermite polynomial used as seed-i. e., the zeros $x_{n}^{\left(\mu_{1}\right)}$ of the polynomials the coefficients of which are the zeros of the Hermite polynomial $H_{N}(z)$. But we also discovered that these matrices were of the following type:

$$
\mathbf{M}^{\left(\mu_{1}\right)}\left(\tilde{x}, \tilde{x}^{\left(\mu_{1}\right)}\right)=\mathbf{R}\left(\tilde{x}^{\left(\mu_{1}\right)}\right) \mathbf{M}(\tilde{x})\left[\mathbf{R}\left(\tilde{x}^{\left(\mu_{1}\right)}\right)\right]^{-1},
$$

so that their property to have the same spectrum as the matrix $\mathbf{M}(\tilde{x})$ implies no special property of the $N$ zeros of the set $\tilde{x}^{\left(\mu_{1}\right)}$; indeed, this conclusion is implied by this formula for any arbitrary definition of the matrix $\mathbf{R}\left(\tilde{x}^{\left(\mu_{1}\right)}\right)$, under the sole condition that this matrix be invertible. And we also convinced ourselves (see Appendix B, where the derivation of the above formula is reported) that - at least via the technique we use there - the same phenomenon would also happen for matrices constructed with the zeros of subsequent generations of polynomials or from the zeros of polynomials generated by different seeds. So the identification of Diophantine, or other, remarkable properties of the zeros of the polynomials belonging to generations of polynomials of the type yielded by the approach described in Section 2 remains an open problem.

\section{Appendix A}

In this Appendix A we report the first three generations of the monic seconddegree polynomials, constructed using the procedure described in Section 2, starting from the generic seed polynomial

$$
p_{2}^{(0)}(z)=z^{2}+b z+c,
$$

where $b$ and $c$ are two arbitrary complex numbers.

Before we proceed, let us note that every complex number $\zeta=\rho \exp (\mathbf{i} \phi)$ where of course $\rho$ is a nonnegative real number and $\phi$ is a real number in the 
range $0 \leq \phi<2 \pi$-has two square roots $\pm r$, where $r=\sqrt{\rho} \exp (\mathbf{i} \phi / 2)$ and, for definiteness, the square root $\sqrt{\rho}$ is nonnegative. Using this fact, we introduce the (generally complex) numbers $r_{0}, r_{11}, r_{12}, r_{21}, r_{22}, r_{23}, r_{24}$ as follows:

$$
\begin{array}{lr}
\sqrt{b^{2}-4 c}= \pm r_{0}, & (27 \mathrm{a}) \\
\sqrt{8 b+2 b^{2}-4 c+8 r_{0}-2 b r_{0}}= \pm r_{11}, & (27 \mathrm{~b}) \\
\sqrt{8 b+2 b^{2}-4 c-8 r_{0}+2 b r_{0}}= \pm r_{12}, & (27 \mathrm{c}) \\
\sqrt{-8 b+4 b^{2}-8 c+24 r_{0}-4 b r_{0}+16 r_{11}+2 b r_{11}-2 r_{0} r_{11}}= \pm r_{21},(27 \mathrm{~d}) \\
\sqrt{-8 b+4 b^{2}-8 c+24 r_{0}-4 b r_{0}-16 r_{11}-2 b r_{11}+2 r_{0} r_{11}}= \pm r_{22},(27 \mathrm{e}) \\
\sqrt{-8 b+4 b^{2}-8 c-24 r_{0}+4 b r_{0}+16 r_{12}+2 b r_{12}+2 r_{0} r_{12}}= \pm r_{23},(27 \mathrm{f}) \\
\sqrt{-8 b+4 b^{2}-8 c-24 r_{0}+4 b r_{0}-16 r_{12}-2 b r_{12}-2 r_{0} r_{12}}= \pm r_{24} .(27 \mathrm{~g})
\end{array}
$$

Using this notation, we display below the polynomials in the first three generations, yielded by the seed polynomial (26).

First generation. The polynomials in the first generation are given by

$$
\begin{aligned}
& p_{2}^{(1)}(z)=z^{2}-\frac{b}{2}(z+1)+\frac{r_{0}}{2}(z-1), \\
& p_{2}^{(2)}(z)=z^{2}-\frac{b}{2}(z+1)-\frac{r_{0}}{2}(z-1) .
\end{aligned}
$$

Second generation. The polynomials in the second generation are given by

$$
\begin{aligned}
& p_{2}^{(1,1)}(z)=z^{2}+\frac{b-r_{0}}{4}(z+1)+\frac{r_{11}}{4}(z-1), \\
& p_{2}^{(1,2)}(z)=z^{2}+\frac{b-r_{0}}{4}(z+1)-\frac{r_{11}}{4}(z-1), \\
& p_{2}^{(2,1)}(z)=z^{2}+\frac{b+r_{0}}{4}(z+1)+\frac{r_{12}}{4}(z-1), \\
& p_{2}^{(2,2)}(z)=z^{2}+\frac{b+r_{0}}{4}(z+1)-\frac{r_{12}}{4}(z-1) .
\end{aligned}
$$


Third generation. The polynomials in the third generation are given by

$$
\begin{aligned}
& p_{2}^{(1,1,1)}(z)=z^{2}+\frac{1}{8}\left(-b+r_{0}-r_{11}\right)(z+1)+\frac{r_{21}}{8}(z-1), \\
& p_{2}^{(1,1,2)}(z)=z^{2}+\frac{1}{8}\left(-b+r_{0}-r_{11}\right)(z+1)-\frac{r_{21}}{8}(z-1), \\
& p_{2}^{(1,2,1)}(z)=z^{2}+\frac{1}{8}\left(-b+r_{0}+r_{11}\right)(z+1)+\frac{r_{22}}{8}(z-1), \\
& p_{2}^{(1,2,2)}(z)=z^{2}+\frac{1}{8}\left(-b+r_{0}+r_{11}\right)(z+1)-\frac{r_{22}}{8}(z-1), \\
& p_{2}^{(2,1,1)}(z)=z^{2}+\frac{1}{8}\left(-b-r_{0}-r_{12}\right)(z+1)+\frac{r_{23}}{8}(z-1), \\
& p_{2}^{(2,1,2)}(z)=z^{2}+\frac{1}{8}\left(-b-r_{0}-r_{12}\right)(z+1)-\frac{r_{23}}{8}(z-1), \\
& p_{2}^{(2,2,1)}(z)=z^{2}+\frac{1}{8}\left(-b-r_{0}+r_{12}\right)(z+1)+\frac{r_{24}}{8}(z-1), \\
& p_{2}^{(2,2,2)}(z)=z^{2}+\frac{1}{8}\left(-b-r_{0}+r_{12}\right)(z+1)-\frac{r_{24}}{8}(z-1) .
\end{aligned}
$$

\section{Appendix B}

In this Appendix B we show that the following, rather standard, approach, does not allow to obtain results for the zeros of the polynomials of the generations yielded by any given polynomial playing the role of the seed.

Take as starting point a solvable dynamical system

$$
\dot{\gamma}_{m}(t)=f_{m}(\vec{\gamma}(t)), \quad \dot{\vec{\gamma}}=\vec{f}(\vec{\gamma}) ;
$$

and assume that this system has the equilibrium configuration

$$
\vec{\gamma}(t)=\vec{\gamma}(0)=\vec{x},
$$

where of course

$$
f_{m}(\vec{x})=0, \quad \vec{f}(\vec{x})=\overrightarrow{0} .
$$

Because this system is solvable, one can control the behavior of its solutions for all time from all initial data. Let us then look at its behavior infinitesimally close to its equilibrium configuration, by setting

$$
\vec{\gamma}(t)=\vec{x}+\varepsilon \vec{w}(t)+\vec{o}(\varepsilon),
$$

implying

$$
\dot{\vec{w}}(t)=\mathbf{F}(\vec{x}) \vec{w}(t),
$$

where the $N \times N$ time-independent matrix $\mathbf{F}(\vec{x})$ is defined componentwise as follows:

$$
F_{n m}(\vec{x})=\left.\frac{\partial f_{n}(\vec{\gamma})}{\partial \gamma_{m}}\right|_{\vec{\gamma}=\vec{x}} .
$$


The behavior of the system in the (infinitesimal, immediate) vicinity of its equilibrium configuration is then characterized by exponentials $\exp \left(\varphi_{m} t\right)$ where the $N$ numbers $\varphi_{m}$ are the $N$ eigenvalues of the $N \times N$ time-independent matrix $\mathbf{F}(\vec{x})$. And comparing this behavior with that of the general solution of the solvable system (31a) one gets information on the values of these eigenvalues $\varphi_{m}$.

For instance in this manner, by taking as starting point the solvable dynamical system

$$
\dot{\gamma}_{m}(t)=\mathbf{i}\left\{\gamma_{m}(t)-\sum_{\ell=1, \ell \neq m}^{N}\left[\gamma_{m}(t)-\gamma_{\ell}(t)\right]^{-1}\right\},
$$

one gets Diophantine properties of the matrices (24) manufactured with the zeros $x_{n}$ of Hermite polynomials,

$$
H_{N}\left(x_{n}\right)=0 \text {, }
$$

since these zeros indeed correspond to the equilibrium configuration of the dynamical system (33) because they satisfy — see for instance [12] [13] [5] - the $N$ algebraic equations

$$
x_{n}=\sum_{\ell=1, \ell \neq n}^{N}\left(x_{n}-x_{\ell}\right)^{-1} .
$$

These Diophantine relations consist in the discovery (see for instance [1] [5]) that the $N \times N$ matrix $\mathbf{M}(\tilde{x})$, see (24), features the $N$ eigenvalues $0,1, \ldots, N-1$.

The next step is to introduce the monic polynomial with coefficients $\gamma_{m}(t)$ and $z \operatorname{eros} \xi_{n}(t)$,

$$
\begin{gathered}
p_{N}(z ; \vec{\gamma}(t) ; \tilde{\xi}(t))=z^{N}+\sum_{m=1}^{N}\left[\gamma_{m}(t) z^{N-m}\right], \\
p_{N}(z ; \vec{\gamma}(t) ; \tilde{\xi}(t))=\prod_{n=1}^{N}\left[z-\xi_{n}(t)\right],
\end{gathered}
$$

and the associated dynamical system provided by the identity (6) together with (33):

$$
\begin{aligned}
& \dot{\xi}=-\mathbf{i}\left[\sum_{\ell=1, \ell \neq n}^{N}\left(\xi_{n}-\xi_{\ell}\right)\right]^{-1} . \\
& \cdot \sum_{m=1}^{N}\left[\xi_{n}^{N-m}\left\{\gamma_{m}-\sum_{\ell=1, \ell \neq m}^{N}\left[\gamma_{m}-\gamma_{\ell}\right]^{-1}\right\}\right],
\end{aligned}
$$

where of course, in the right hand side, the coefficients $\gamma_{n} \equiv \gamma_{n}(t)$ should be replaced by their expressions in terms of the zeros $\xi_{n} \equiv \xi_{n}(t)$,

$$
\gamma_{m}=(-1)^{m} \sigma(\tilde{\xi}) \text {. }
$$


We look then at the time evolution of the solutions $\xi_{n}(t)$ of this dynamical system in the immediate vicinity of its equilibrium configuration $\gamma_{m}(t)=$

$\gamma_{m}(0)=x_{m}, \xi_{n}(t)=\xi_{n}(0)=x_{n}^{\left(\mu_{1}\right)}$. It is then easily seen, by setting, together with (32a),

$$
\xi_{n}(t)=x_{n}^{\left(\mu_{1}\right)}+\varepsilon v_{n}(t), \quad \tilde{\xi}(t)=\tilde{x}^{\left(\mu_{1}\right)}+\varepsilon \tilde{v}(t)
$$

and by proceeding as above, that one arrives at the linear evolution system

$$
\dot{\tilde{v}}=\mathbf{M}^{\left(\mu_{1}\right)}\left(\tilde{x}, \tilde{x}^{\left(\mu_{1}\right)}\right) \tilde{v}
$$

where the $N \times N$ matrices $\mathbf{M}^{\left(\mu_{1}\right)}\left(\tilde{x}, \tilde{x}^{\left(\mu_{1}\right)}\right)$ are defined by (25) (with (24) and of course the matrices $\mathbf{R}(\tilde{x})$ and $[\mathbf{R}(\tilde{\mathbf{x}})]^{-\mathbf{1}}$ defined as above, see (15)), the numbers $x_{n}$ are the $N$ zeros of the Hermite polynomial $H_{N}(z)$ of order $N$ and the numbers $x_{n}^{\left(\mu_{1}\right)}$ are the $N$ zeros of the (monic) polynomials the coefficients of which are the zeros of the Hermite polynomial $H_{N}(z)$ ordered according to the their permutation $\mu_{1}$.

It is plain from the treatment sketched in this Appendix B that the statements reported in the last part of Section 4 are validated.

\section{References}

[1] Ahmed, S., Bruschi, M., Calogero, F., Olshanetsky, M.A. and Perelomov, A.M.: Properties of the zeros of the classical polynomials and of Bessel functions, Nuovo Cimento 49B 173-199 (1979).

[2] Bihun, O. and Calogero, F.: New solvable many-body problems of goldfish type, J. Nonlinear Math. Phys. (in press), arXiv:1507.03959 [math-ph].

[3] Calogero, F.: Motion of Poles and Zeros of Special Solutions of Nonlinear and Linear Partial Differential Equations, and Related "Solvable" Many Body Problems, Nuovo Cimento 43B 177-241 (1978).

[4] Calogero F.: The "neatest" many-body problem amenable to exact treatments (a "goldfish"?), Physica D 152-153 78-84 (2001).

[5] Calogero, F.: Classical many-body problems amenable to exact treatments, Lecture Notes in Physics Monographs m66, Springer, Heidelberg (2001).

[6] Calogero, F.: Isochronous systems, Oxford University Press, (2008); marginally updated paperback edition (2012).

[7] Calogero, F.: New solvable variants of the goldfish many-body problem, Studies Appl. Math. (in press; published online 07.10.2015). 
[8] Calogero, F.: A solvable $N$-body problem of goldfish type featuring $N^{2}$ arbitrary coupling constants, J. Phys. A: Math. Theor. (submitted to, 18.09.2015).

[9] Calogero, F. and Gómez-Ullate, D.: Asymptotically isochronous systems, J. Nonlinear Math. Phys.15 410-426 (2008).

[10] Erdélyi, A. (editor): Higher transcendental functions, vol. II, McGraw-Hill, New York (1953).

[11] Gómez-Ullate, D. and Sommacal, M.: Periods of the goldfish many-body problem, J. Nonlinear Math. Phys. 12, Suppl. 1 351-362 (2005).

[12] Stieltjes, T. J.: Sur certains polynómes qui vérifient une équation différentielle linéaire du second ordre et sur la théorie des fonctions de Lamé, Acta Math. 8, 321-326 (1885); Oeuvres Complètes 1, 434-439.

[13] Szegő, G.: Orthogonal Polynomials (4th Edition), American Mathematical Society, Providence, RI (1975). 